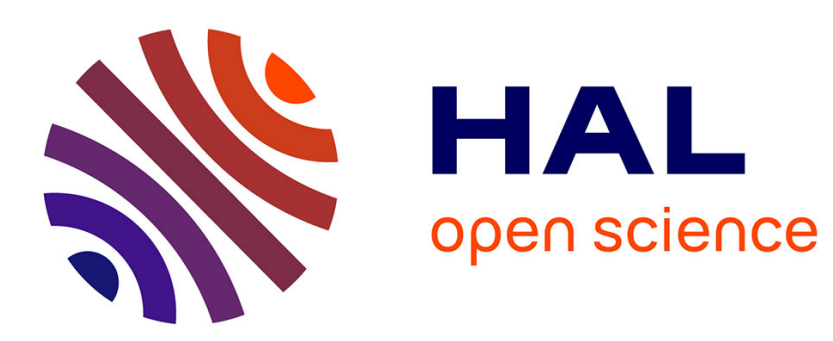

\title{
On a Hamilton-Jacobi-Bellman approach for coordinated optimal aircraft trajectories planning
}

Céline Parzani, Stéphane Puechmorel

\section{To cite this version:}

Céline Parzani, Stéphane Puechmorel. On a Hamilton-Jacobi-Bellman approach for coordinated optimal aircraft trajectories planning. Optimal Control Applications and Methods, 2017, Special Issue: Global and robust optimization of dynamic systems, 39 (2), pp.933-948. 10.1002/oca.2389 . hal01340565

\section{HAL Id: hal-01340565 \\ https://hal-enac.archives-ouvertes.fr/hal-01340565}

Submitted on 1 Jul 2016

HAL is a multi-disciplinary open access archive for the deposit and dissemination of scientific research documents, whether they are published or not. The documents may come from teaching and research institutions in France or abroad, or from public or private research centers.
L'archive ouverte pluridisciplinaire HAL, est destinée au dépôt et à la diffusion de documents scientifiques de niveau recherche, publiés ou non, émanant des établissements d'enseignement et de recherche français ou étrangers, des laboratoires publics ou privés. 


\title{
On a Hamilton-Jacobi-Bellman approach for coordinated optimal aircraft trajectories planning
}

\author{
Céline Parzani, Stéphane Puechmorel \\ Universit Fédérale de Toulouse \\ Ecole Nationale de l'Aviation Civile \\ MAIAA \\ F-31055 Toulouse, FRANCE
}

\begin{abstract}
In the context of future air traffic management (ATM), an increasing importance is given to environmental considerations and especially fuel consumption. It is thus advisable to make an optimal use of external conditions knowledge, like wind or temperature, to reduce the total fuel needed to complete a flight. On the other hand, safety must be guaranteed all over the trajectory: encounters below the regulatory separation minima, termed as conflicts, must never occur. In this paper, we consider the problem of optimally planning conflict free aircraft trajectories, based on a minimal time criterion and taking into account an ambient wind field. Aircraft motions are restricted to the horizontal plane only in order to reduce the dimension of the problem and to avoid costly changes of flight level. Since global optimality for the whole set of aircraft is sought after, the admissible space is modeled after a Cartesian product of the individual, two-dimensional state spaces with forbidden configurations removed. An Hamilton-Jacobi-Bellman approach in the so-called configuration space obtained that way is then applied to get a coordinated, conflict-free optimal planning. One of the main advantages of this method is the insurance of getting a global optimum without having to rely on an complex initialization procedure. However, as the size of the instances increases with the power of the number of aircraft considered, a dedicated numerical algorithm must be designed. In this work, the Ultra-Bee scheme is adapted to configuration spaces and solved in a four dimensional plus time setting.
\end{abstract}

Keywords: Hamilton-Jacobi-Bellman, Aircraft trajectory coordinated planning, high dimension

\section{Introduction}

Aircraft trajectory optimization while ensuring flights safety is, from the operational point of view, a subject of great importance in air traffic management (ATM). It becomes more and more challenging with the expected growth of air traffic for the next 20 years.

As a consequence, many ATM applications aiming at improving the performance of the overall system involve optimal aircraft trajectories planning. This question deals with the generation of a path from departure to destination taking into account several objectives (minimizing distance, motion time or fuel) while avoiding obstacles and complying with aircraft dynamics.

A survey of mathematical models for aircraft trajectory design can be found in [20]. Planning several 10 trajectories interacting with each other is a complex problem and different approaches can be considered to 
solve it. Among them, the simplest and most common one is the sequential approach. It consists first in computing the optimal trajectory for a given mobile. Next, the trajectory for a second mobile is computed considering the previous one as a moving obstacle and so on. It has been used for instance in [23, 26, 35]. The two main drawbacks are that a admissible solution may not be found even if one exists and second that 15 a global optimum may not be reached. A second class concerns iterative method (see [15]) and consists in first planning individual trajectories, then identifying potential conflicts and finally using metaheuristics like genetic algorithms. This method can reach an asymptotically optimal solution. However, for a given computing time, a feasible (conflict-free) solution is not guaranteed to be reached. A third class of methods is the Model Predictive Control (see for instance [32]) which consists in solving at each time step an optimal control problem to go from the current state to the target state satisfying the constraints and then finding the optimal control for each aircraft minimizing the sum, over the whole set of aircraft, of individual cost functions. Moreover, an approach based on Multi-Agents System has also been explored in [30,33]. The main drawback is that emergence of the expected complex behavior is not always guaranted. Last, we find the coordinated motion approach which takes into account the whole set of mobiles simultaneously leading to more complex methods. Among deterministic methods, we can cite some works based on navigation functions (see [27]). Moreover, in [1], Alton optimizes the trajectory of two robots without collision. Instead of solving the problem in $\mathbb{R}^{2}$, he worked in the configuration space of several robots. For instance, for two robots, the work space is constructed removing from $\mathbb{R}^{4}$ the collision points between robots. In our case, the mathematical definition of the configuration space will be given in Section 2.1.

which is defined such that the collision points between robots are excluded from the space. Computed trajectories are then ensured to be without collision. Space dimension increasing exponentially is the main drawback of these algorithms.

In this context, the objective of this paper is to propose a new framework for trajectories planning with coordinated motion ensuring the following key points. First, we are looking for a global solution for simultaneous planning several aircraft trajectories. On the other hand, the model has to be able to avoid collisions and to take into account external components such as wind fields.

To reach our objective, we investigate an optimal control approach. First introduced in [12], it has been widely used in litterature for trajectories planning for which it is well-suited. The objective of such approach is to determine the control inputs such that the response of a dynamical system satisfies the physical constraints, while, at the same time, minimizes some performance criterion. Aircraft trajectory optimization can then be considered as an optimal control problem in which the control variables are time varying. Trajectory feasibility is automatically ensured and reconstruction is then performed from the optimal value of the control at each time step.

Among the methods to solve optimal control problems, we will consider in this work, the Hamilton-Jacobi45 Bellman approach. Based on the Dynamic Programming Principle [3], it can be used to solve fully nonlinear control problems and minimum time problems, taking into account mixed state/control constraints (see [2]). On the opposite of Direct Approach [4] or Pontryagin-based optimization algorithms that rely on necessary condition for optimality, HJB approach gives a global optimum avoiding initialization and convergence questions. 
For instance, according to [20], the optimal control formulation of a trajectory optimization problem using

50 Pontryagin's Maximum Principle leads to a Two-point Boundary Value Problem. Numerical techniques such as shooting methods can be applied to solve accurately this Two-point Boundary Value Problem, but their convergence is very sensitive to the choice of an initial guess for the solution. Moreover, an interesting byproduct of the HJB approach is the synthesis of the optimal control in feedback form. Once the HJB equation is solved, for any starting point, the reconstruction of the optimal trajectory can be performed in real time. In return, the method has to face the so-called curse of dimensionality which implies challenging development in numerical methods to make it amenable to implementation.

HJB framework has already been used for trajectories planning (see for instance $[9,10,19]$ ). In this paper, the aim is to demonstrate the potential of HJB approach to plan trajectories for coordinated motion in the presence of wind. Let us note that when wind is taken into account and has a non negligible speed with respect 60 to aircraft, the problem is no longer isotropic. Compared with existing works, the main contribution of this paper is to derive a model able to take into account several mobiles moving simultaneously while the wind effect is described through the dynamic of the system using some control parameters. Moreover, we show how to take advantage of the mathematical formalism to describe conflict avoidance solving HJB system in the configuration space.

In order to evaluate the relevance of our approach, we consider in this study a scenario with only two aircraft. The model is derived following the formalism proposed in [5] and it leads to the resolution of the HJB equations in a four dimensional space (2D for each aircraft) plus time. Since the aim is to evaluate the HJB approach in term of modeling, we focus on the precision of its numerical solution. Indeed, in order to be able to describe phenomena such as conflict avoidance or obstacles bypassing, the numerical scheme used must provide an accurate description of a front expansion problem. Therefore, we propose to solve the 4D HJB system with the anti-diffusive UltraBee scheme first developed in [21, 22]. The CPU time reduction particularly interesting when considering more than two aircraft with an operational objective will be studied in a further work taking advantage of recent numerical developments.

The paper is organized as follows. In Section 2, we first describe the optimal control problem for two aircraft 75 in a wind field. Next, we present the formalism leading to the Hamilton-Jacobi-Bellman system for the problem we are interested in. In Section 3, we present the numerical methods used to solve the problem. First, we recall the Ultra-Bee scheme for the 4-dimensional space case with obstacles. Then, the algorithm for trajectory reconstruction is presented. Numerical experiments showing the relevance of this approach are performed in Section 4 for a two aircraft conflict avoidance scenario. Finally, conclusions of the work and directions for further works are given in Section 5.

\section{Hamilton-Jacobi-Bellman approach for two aircraft trajectories planning}

This section aims at describing the physical problem and its mathematical formulation. We are interested in solving a minimum-time path problem for two aircraft under the following assumptions. The two mobiles are moving in a two dimensional space (planar cartesian positions) and aircraft are cruising at a fixed altitude.

${ }_{85}$ Their speed is assumed to be constant and they are only controlled by the heading direction. The objective 
is then to plan the optimal paths avoiding obstacles and taking into account wind currents. The approach developed in the following section is based on a complex control optimal problem in high dimension solved using HJB equations. This technique has already been studied for trajectories planning (see $[9,10,19,28])$ and to formulate the model we will follow the mathematical framework proposed in $[5,6]$. The main contribution of this work is to adapt existing works to propose a model able to manage several mobiles taking into account conflict avoidance in the HJB framework. The technique presented in this paper is based on the computation of the backward reachable set.

It consists in computing the set of initial departure position from which aircraft can reach its destination the target - avoiding conflicts with other aircraft and obstacles within a given time $t \geq 0$. The model description 95 only concerns two aircraft in the following paragraph but it can be extended to a higher number of mobiles. This specific aspect of the problem requires a special numerical treatment and will be addressed in a separate work.

\subsection{Optimal control problem formulation}

Let us consider two aircraft $M_{1}$ and $M_{2}$ at a given flight level during cruise phase. For $i=1,2$, suppose that at any time $t$, the position of aircraft $M_{i}$ is a vector $X_{i}(t) \in \mathbb{R}^{2}$. The optimal control problem consists in computing the optimal coordinated control for the system composed by the two aircraft in order to minimize a criterion.

Let us first define the state variables. In our case, they will correspond to the planar cartesian coordinates of the aircraft $M_{1}$ (respectively $M_{2}$ ) denoted $X_{1}(t)$ (respectively $X_{2}(t)$ ). Let us note that in this study the initial positions of aircraft as well as the final ones are known.

Resulting from aircraft's dynamics, these state variables are subject to dynamical laws yielding to differential equations on position. For $i=1,2$ let $A_{i}$ be a nonempty compact subset of $\mathbb{R}$. Let $f_{i}: \mathbb{R}^{2} \times A_{i} \mapsto \mathbb{R}^{2}$ be Lipschitz continuous with respect to $\left(X_{i}, a_{i}\right)$. Moreover, for each aircraft $i=1,2$, we define the set of controls $\mathcal{A}_{i}$

$$
\mathcal{A}_{i}:=\left\{a_{i}: \mathbb{R}^{+} \mapsto A_{i}, a_{i} \text { measurable }\right\}
$$

Given an initial state $X_{i}^{0}$ corresponding to departure position for aircraft $M_{i}$ and $i=1,2$, the dynamic of the system is given by

$$
\left\{\begin{array}{l}
\dot{X}_{1}(t)=f_{1}\left(X_{1}(t), a_{1}(t)\right) \quad t \geq 0 \\
\dot{X}_{2}(t)=f_{2}\left(X_{2}(t), a_{2}(t)\right) \quad t \geq 0 \\
\left(X_{1}(0), X_{2}(0)\right)=\left(X_{1}^{0}, X_{2}^{0}\right) \in \mathbb{R}^{4}
\end{array}\right.
$$

where the controls $a_{1} \in \mathcal{A}_{1}, a_{2} \in \mathcal{A}_{2}$ must be determined later according to conflict avoidance rules.

In particular, when we consider wind currents, for each aircraft $M_{i}$ with position $X_{i}(t)=\left(x_{i}, y_{i}\right) \in \mathbb{R}^{2}$ at

time $t$, the dynamic of the system is defined as follows:

$$
\dot{X}_{i}(t)=V h\left(a_{i}(t)\right)+W_{i}\left(t, X_{i}(t)\right)
$$

where $V$ is the constant speed of the aircraft, $W$ is the wind field vector and $a_{i} \in \mathcal{A}_{i}$ is the control parameter of the optimal path corresponding in our case to the heading angle relative to North direction for aircraft $M_{i}$. 
Here the admissible control set is $A_{i}=[0,2 \pi]$ and the associated function $h$ is defined by

$$
\begin{aligned}
h: & {[0,2 \pi[\mapsto \mathbb{R} \times \mathbb{R}} \\
& \theta \rightarrow(\cos (\theta), \sin (\theta)) .
\end{aligned}
$$

The vector $W=(u, v)$ is given such that $u$ is the east component of the wind, and $v$ is the north component of

Let us note that in this study, we assume that during cruise phase, aicrafts are moving at a given optimal flight level. Therefore, the vertical component for wind is not taken into account.

In the sequel, we first define the set of controls $\mathcal{A}=\mathcal{A}_{1} \times \mathcal{A}_{2}$ and the state variable $Z=\left(X_{1}, X_{2}\right) \in \mathbb{R}^{4}$. Then, for any control value $a \in \mathcal{A}$, the function $Z$ is solution of the system

$$
\left\{\begin{array}{l}
\dot{Z}(t)=f(Z(t), a(t)) \quad t \geq 0, \\
Z(0)=z^{0}:=\left(X_{1}^{0}, X_{2}^{0}\right) .
\end{array}\right.
$$

Furthermore, for any control value $a \in \mathcal{A}$, we denote the set of associated trajectories on $[0, T]$ by

$$
\mathcal{K}_{a}=\left\{Z(t) \in \mathbb{R}^{4}, \text { with } t \in[0, T], Z \text { satisfies system }(2.3)\right\}
$$

leading to the whole set of trajectories denoted $\mathcal{K}:=\bigcup_{a \in \mathcal{A}} \mathcal{K}_{a}$.

Now we define the state-constraints set that allows to model both conflict avoidance between aircraft as well as fixed obstacles bypassing.

${ }_{25}$ Definition 2.1 (conflict avoidance). We say that two aircraft $M_{1}$ and $M_{2}$ are in a "conflict avoidance" configuration if the distance between the two at any time is larger than a safety value $\epsilon>0$. Hence we define $\mathcal{C}_{1}^{\varepsilon} \subset \mathbb{R}^{4}$ as

$$
\mathcal{C}_{1}^{\varepsilon}=\left\{Z=\left(X_{1}, X_{2}\right) \in \mathbb{R}^{4}: d\left(X_{1}, X_{2}\right)>\varepsilon\right\}
$$

the subset of $\mathbb{R}^{4}$ in which conflicts may not occur.

Definition 2.2 (fixed obstacles bypassing). Let us denote $\mathcal{O} \subset \mathbb{R}^{4}$ a set of fixed obstacles then we define $\mathcal{C}_{2}^{\varepsilon}$ the set of constraints enforcing the aircraft to bypass the set of obstacles by

$$
\mathcal{C}_{2}^{\varepsilon}=\left\{Z \in \mathbb{R}^{4} ; d\left(X_{1}, \mathcal{O}_{1}\right)>\varepsilon, d\left(X_{2}, \mathcal{O}_{2}\right)>\varepsilon \text { with }\left(\mathcal{O}_{1}, \mathcal{O}_{2}\right) \in \mathcal{O}\right\}
$$

We are now able to define the set where the admissible trajectories of aircraft lie. For instance in the case where the aim is to take into account conflict avoidance and fixed obstacles in the model, we have:

Definition 2.3. A trajectory $\mathcal{S}:=\{Z(t) ; t \in[0, T]\}$ will be "admissible" if $Z$ is solution of (2.3) and satifies the state-constraints on the time interval $[0, T]$, that means

$$
\mathcal{S} \in \mathcal{K} \cap\left(\mathcal{C}_{1}^{\varepsilon} \cup \mathcal{C}_{2}^{\varepsilon}\right)
$$

In this work, we aim at planning aircraft trajectories from departure to arrival positions which corresponds to a front propagation problem for the optimal control problem we consider. Therefore, we will introduce the target $\mathcal{C}$ a nonempty closed set of $\mathbb{R}^{4}$ modeling arrival positions and representing the front at time $t=0$. For numerical implementation, we will assume that $\mathcal{C}$ is bounded. We have the following definition 
Definition 2.4. The capture basin $C a p_{\mathcal{C}}^{f}(t)$ is the the set of initial points $z^{0} \in \mathbb{R}^{4}$ from which starts an admissible trajectory $\{Z(s) ; s \in[0, t]\}$ solution of (2.3) associated to an admissible control $a \in \mathcal{A}$ and such that $Z$ reaches the target $\mathcal{C}$ at time $t \geq 0$

Remark 2.5. Let us note that for all $t \geq 0$, the set $C a p_{\mathcal{C}}^{f}(t)$ contains the initial positions which can be steered to the target exactly at time $t$.

We also define the backward reachable set associated to $\mathcal{C}$ for the dynamic $f$ by

Definition 2.6. The backward reachable $\operatorname{set} \mathcal{R}_{\mathcal{C}}^{f}([0, t])$ associated to the target $\mathcal{C}$ is the set of all initial conditions $z^{0} \in \mathbb{R}^{4}$ from which it is possible to find an admissible trajectory $\{Z(s) ; s \in[0, t]\}$ where $Z$ is solution of (2.3) reaching the target $\mathcal{C}$ before time $t \geq 0$ starting from $z^{0}$.

In this work, the objective is to reduce costs associated to cruise phase. These costs mainly depend on two correlated parameters: consumption and flight duration. Let us denote by $\mathcal{T}$ the cost to optimize. From the Bolza formulation and for a free final time problem $T$, the cost functional can be written as

$$
\mathcal{T}\left(z^{0}, a\right):=\int_{0}^{T} g(t, Z(t), a(t)) d t+l(T, Z(T)) .
$$

where $g$ represents the instantaneous cost and $l$ represents the cost associated to the final position.

The control problem we are interested in can then be written as follows: for a given $z^{0} \in \mathbb{R}^{4}$, we look for $a^{\star} \in \mathcal{A}, Z^{\star}$ such that $Z^{\star}(0)=z^{0}, Z^{\star}(T) \in \mathcal{C}$ and $\left\{Z^{\star}(t), t \in[0, T]\right\} \subset \mathcal{K}_{a^{\star}} \cap\left(\mathcal{C}_{1}^{\varepsilon} \cup \mathcal{C}_{2}^{\varepsilon}\right)$ where $a \star$ is solution to

$$
\mathcal{T}\left(z^{0}, a^{\star}\right):=\min _{a \in \mathcal{A}} \mathcal{T}\left(z^{0}, a\right)
$$

Remark 2.7. Let us note that the formulation proposed for problem (2.8) ensure to get the minimal cost for each aircraft. Indeed, the only coupling condition between the two mobiles is expressed through the admissible space $\mathcal{K}$ which can be viewed as a forbidden area for the two aircraft. As a consequence, the optimal trajectory for each aircraft can easily be deduced from the $4 D$ trajectory reconstructed from the solution of problem (2.8).

\subsection{Hamilton-Jacobi-Bellman equation}

Assuming that the speed aircraft is constant, the consumption mainly depends on time flight. Hence, neglecting the over-consumption resulting from changes of direction, minimizing time flight leads to minimizing consumption. As a consequence, the criterion to optimize in our study will be the time flight. Therefore, letting $g$ equal to 1 and $l$ equal to 0 in (2.7), the cost $\mathcal{T}$ is now the minimal time for each aircraft needed to reach the destination $\mathcal{C}$ from the departure position $z^{0}=\left(X_{1}^{0}, X_{2}^{0}\right) \in \mathbb{R}^{4}$ with an admissible trajectory obeying the state/control constraints.

The optimization problem then only consists in finding the best control $a^{\star}(t) \in \mathcal{A}$ for $t \in[0, T]$ to minimize the total travel time.

In order to solve problem (2.8) on $\mathbb{R}^{2} \times \mathbb{R}^{2}$, we will use the Hamilton-Jacobi-Bellmann approach which consists in proving that $\mathcal{T}$ is a solution of a nonlinear time evolution Hamilton Jacobi partial differential equation set in $(t, Z(t)) \in] 0, \infty\left[\times \mathbb{R}^{4}\right.$. 
The form of the Hamilton-Jacobi equation is based on Bellman's optimality principle in discrete case [3]. The resolution of this Partial Differential Equation on the whole space gives a necessary and sufficient condition for optimality. This is a main advantage of the methodology developed in this paper compared to others methods that need suitable initialization and convergence study. It can be seen as a front expansion problem where the wave front represents the minimum time to reach the arrival point. The wave front is computed gradually from the previous front positions.

In order to compute the function $\mathcal{T}$, we will focus on computation of backward reachable sets associated to the target $\mathcal{C}$ for the dynamic $f$ of the problem (2.3). In [29], the authors proved that that the viscosity solution of a particular time-dependent Hamilton Jacobi Isaacs PDE provides an implicit surface representation of the continuous backwards reachable set. From these results (see also [5]), we have the following proposition:

Proposition 2.8. Introducing a new virtual control $\lambda($.$) with \forall s \in[0, t] \lambda(s) \in[0,1]$, the backward reachable set 180 $\mathcal{R}_{\mathcal{C}}^{f}([0, t])$ defined by (2.6) is exactly the capture basin $\operatorname{Cap}_{\mathcal{C}}^{\tilde{f}}(t)$ associated to $\mathcal{C}$ and the dynamic $\tilde{f}\left(z^{0},(a, \lambda):=\right.$ $\lambda f\left(z^{0}, a\right)$ for all $(a, \lambda) \in \tilde{\mathcal{A}}=\mathcal{A} \times[0,1]$.

We will take advantage of this property to compute the minimum time $\mathcal{T}$. Let now $\phi: \mathbb{R}^{4} \rightarrow \mathbb{R}$ be Lipschitz continuous and a level set function for the target that means :

$$
\phi(Z(t)) \leq 0 \Longleftrightarrow Z(t) \in \mathcal{C}
$$

We also introduced $g$ a level set function associated to the state-constraints $\mathcal{K} \cap\left(\mathcal{C}_{1}^{\varepsilon} \cup \mathcal{C}_{2}^{\varepsilon}\right)$ such that $g: \mathbb{R}^{4} \rightarrow \mathbb{R}$ Lipschtiz continuous and

$$
g(Z(s), s) \leq 0 \Longleftrightarrow Z(s) \in \mathcal{K} \cap\left(\mathcal{C}_{1}^{\varepsilon} \cup \mathcal{C}_{2}^{\varepsilon}\right) \quad \forall s \in[0, T], Z(s) \in \mathbb{R}^{4}
$$

We now consider the value function $v$ defined by

$$
v\left(z^{0}, t\right)=\inf _{a \in \mathcal{A}} \max \left(\phi(Z(t)), \max _{s \in[0, t]} g(Z(s))\right.
$$

Then it can be proven (see [5]) that this function $v$ is a level set function for the capture basin $\operatorname{Cap}_{\mathcal{C}}^{\tilde{f}}(t)$ such that

$$
\operatorname{Cap}_{\mathcal{C}}^{\tilde{f}}(t)=\left\{z^{0} \in \mathbb{R}^{4} \mid v\left(z^{0}, t\right) \leq 0\right\}
$$

Moreover, we have the following result (see [5])

Proposition 2.9. The value function $v$ is the unique continuous viscosity solution (in the sense of [2]) of the following Hamilton-Jacobi-Bellman (HJB) equation

$$
\left\{\begin{array}{l}
\min \left(\partial_{t} v+\mathcal{H}\left(Z(t), \nabla_{Z} v\right) ; v-g(Z(t))\right)=0 \quad t>0, Z(t) \in \mathbb{R}^{4} \\
v(Z(t), 0)=\max (\phi(Z(t)), g(Z(t))), \quad Z(t) \in \mathbb{R}^{4}
\end{array}\right.
$$

where the Hamiltonian is given by

$$
\mathcal{H}\left(Z(t), \nabla_{Z} v\right):=\max _{\tilde{a} \in \tilde{\mathcal{A}}}(-\tilde{f}(Z(t), \tilde{a}) \cdot p) \quad p \in \mathbb{R}^{4}
$$


Let us now detail the computation of the minimum time function $\mathcal{T}$ we aim to minimize and defined by (2.8). From [5], it can be shown that

$$
\mathcal{T}\left(z^{0}, a^{\star}\right)=\inf t \in[0, T], v\left(z^{0}, t\right) \leq 0 .
$$

Afterwards, the minimal time function is used to recover the optimal control and optimal trajectories from any point of the state space.

\section{Numerical resolution}

\subsection{Ultra-Bee scheme for HJB resolution}

In this section, we are interested in the numerical approximation of the first order Hamilton Jacobi Bellman (HJB) equation resulting from aircraft trajectories planning given by (2.9)-(2.10). A large variety of numerical methods have been studied to determine approximations of the viscosity solutions of systems of type (2.9)-(2.10) which remains a challenging problem, in particular in high dimensions.

Some of them deal with finite difference methods as the first order monotone schemes proposed in [18] or higher ones (ENO scheme in [31] and WENO scheme in [34]). A second class of methods concerns Discontinuous Galerkin methods, a direct method was proposed in [16] and a scheme for front propagation with obstacles in [11]. Then, we can also consider Semi-Lagrangian schemes which are based on the discretization of the dynamic programming principle as developed in $[24,25]$ and [14] for instance. A brief review of different efficient techniques that have been proposed can also be found in [13].

In this paper, we use the Ultra Bee scheme (a finite difference type scheme) to solve the HJB equations (2.9)-(2.10). First developed for advection equations with constant velocity [21, 22], a generalization to velocity with changing sign is suggested in [7] and the adaptation to solve HJB equations is done in [8]. Due to very good anti-diffusive properties, this scheme allows computations of the reachable sets with good accuracy and therefore to get a precise localization of the discontinuity. Indeed, the problem we are interested in can be viewed as a front expansion problem where the wave front represents the minimum time to reach a position, which corresponds to a discontinuity from numerical point of view.

Let us now recall the algorithm applied to discretize the problem (2.9)-(2.10). Let $\mathcal{D}=\left[X_{1, \min }, X_{2, \min }\right] \times$ $\left[Y_{1, \min }, Y_{2, \min }\right]$ be a bounded square box corresponding to the discrete computational domain. We consider a regular spatial grid $\mathcal{G}$ of $\mathcal{D} \subset \mathbb{R}^{4}$ defined by the constant mesh size $\Delta x$ in all directions. Then, for $I=$ $\{i, j, k, l\} \in \mathbb{Z}^{4}$, let

$$
\xi_{I}:=(i \Delta x, j \Delta x, k \Delta x, l \Delta x)
$$

be the center of the cell

$$
M_{I}=\left[x_{1, i-\frac{1}{2}}, x_{1, i+\frac{1}{2}}\right] \times\left[y_{1, j-\frac{1}{2}}, y_{1, j+\frac{1}{2}}\right] \times\left[x_{2, k-\frac{1}{2}}, x_{2, k+\frac{1}{2}}\right] \times\left[y_{2, l-\frac{1}{2}}, y_{2, l+\frac{1}{2}}\right]
$$

We solve the HJB equation on the time interval $\left[0, T_{\max }\right]$. Let us denote $\Delta t$ the time step and for $n \geq 0$, $t^{n}:=n \Delta t$. The following steps are applied. 
Let us first starts by the initialization step. The finite-volume like UltraBee scheme aims at computing a numerical approximation $V_{I}^{n}$ of the mean value of $v$ solution of (2.9)-(2.10) on cell $M_{I}$ at time $t^{n}$. Thus,

$$
V_{I}^{n} \approx \frac{1}{m\left(M_{I}\right)} \int_{M_{I}} v(t, \zeta) d \zeta
$$

The initialization is then done using the average values of $\varphi$ :

$$
V_{I}^{0}=\frac{1}{m\left(M_{I}\right)} \int_{M_{I}} \varphi(\zeta) d \zeta
$$

the average values of $g$ on the grid are computed following

$$
W_{I}=\frac{1}{m\left(M_{I}\right)} \int_{M_{I}} g(\zeta) d \zeta
$$

and we consider an approximation $\mathcal{S}$ of $\lambda$ such that for $\zeta \in \mathcal{D}$

$$
\mathcal{S}(\zeta)= \begin{cases}\{1\} & \text { if } \zeta \notin \mathcal{C}, \\ \{0,1\} & \text { if } \zeta \in \mathcal{C}\end{cases}
$$

We recall that $\mathcal{C}$ is a non empty closed set of $\mathbb{R}^{4}$ and represents aircraft destination in our application.

The next step consists in the set controls discretization.

${ }_{225}$ The set of controls $\mathcal{A}=\mathcal{A}_{1} \times \mathcal{A}_{2}$ is discretized using $N_{1} N_{2}$ values and we set

$$
\mathcal{A}^{d}:=\left\{a_{1}^{1}, \ldots, a_{1}^{N_{1}}\right\} \times\left\{a_{2}^{1}, \ldots, a_{2}^{N_{2}}\right\} .
$$

Now, let us detail the time evolution step of the algorithm. For $n \geq 1$, let us assume that $V_{I}^{n}$, solution of (2.9)-(2.10) on cell $M_{I}$ at time $t^{n}$, is known. Moreover, for $a^{d} \in \mathcal{A}^{d}$ and $\lambda^{d}$ an approximation of $\lambda\left(\xi_{I}\right)$, let us denote $U_{I}^{n+1}\left(a^{d}, \lambda^{d}\right)$ the numerical solution of the advection equation

$$
\frac{\partial u}{\partial t}(t, Z(t))-\lambda(Z(t)) f(t, a) \cdot \nabla_{Z} u(t, Z(t))=0 .
$$

The solution of this advection problem is obtained using the Ultra-Bee Scheme. Since 4 dimensions have to be considered ( $2 D$ for each mobile) in our study, we will use a classical Trotter splitting or "alternate direction method" such that the numerical solution evolves during a time step in each direction. The resolution using this splitting technique is stable under the following CFL condition,

$$
\max _{I, a}\left(\max \left(\frac{\Delta t}{\Delta x}\left|f_{1}\left(\xi_{I}, a_{1}\right)\right|, \frac{\Delta t}{\Delta x}\left|f_{2}\left(\xi_{I}, a_{2}\right)\right|\right)\right) \leq 1
$$

For sake of simplicity, let us present the UltraBee scheme only for the first step of the splitting corresponding to the $x_{1}$-direction. Thus, $\forall i \in \mathbb{Z}$ and for $(j, k, l)$ fixed in $\mathbb{Z}^{3}$, let us note $U_{i, .}^{n}=U_{I}^{n}$, then we have

$$
U_{i, .}^{n+1}\left(a^{d}, \lambda^{d}\right)=U_{i, .}^{n}-\frac{\Delta t}{\Delta x} f_{1}\left(\xi_{I}, a_{1}\right)\left(F_{i+\frac{1}{2}}^{L}-F_{i-\frac{1}{2}}^{R}\right)
$$

Let us denote by $\nu_{i}=\frac{\Delta t}{\Delta x} f_{1}\left(\xi_{I}, a_{1}\right)$ and define the "fluxes" $F_{i+\frac{1}{2}}^{L}$ and $F_{i-\frac{1}{2}}^{R}$. Following the extension of the original scheme in the case where the velocity is changing of sign proposed in [7], they are given by 
1. if $\nu_{i}>0, F_{i+\frac{1}{2}}^{L}=\min \left(\max \left(U_{i+1, .}^{n}, b_{i}^{n,+}\right), B_{i}^{n,+}\right)$ where $b_{i}^{n,+}$ and $B_{i}^{n,+}$ are defined by

$$
\left\{\begin{array}{l}
b_{i}^{n,+}=\max \left(U_{i, .}^{n}, U_{i-1, .}^{n}\right)+\frac{1}{\nu_{i}}\left(U_{i, .}^{n}-\max \left(U_{i, .}^{n}, U_{i-1, .}^{n}\right)\right), \\
B_{i}^{n,+}=\min \left(U_{i, .}^{n}, U_{i-1, .}^{n}\right)+\frac{1}{\nu_{i, .}}\left(U_{i, .}^{n}-\min \left(U_{i, .}^{n}, U_{i-1, .}^{n}\right)\right)
\end{array}\right.
$$

2. if $\nu_{i}<0, F_{i-\frac{1}{2}}^{R}=\min \left(\max \left(U_{i-1, .}^{n}, b_{i}^{n,-}\right), B_{i}^{n,-}\right)$ with

$$
\left\{\begin{array}{l}
b_{i}^{n,-}=\max \left(U_{i, .}^{n}, U_{i+1, .}^{n}\right)+\frac{1}{\left|\nu_{i}\right|}\left(U_{i, .}^{n}-\max \left(U_{i, .}^{n}, U_{i+1, .}^{n}\right)\right), \\
B_{i}^{n,-}=\min \left(U_{i, .}^{n}, U_{i+1, .}^{n}\right)+\frac{1}{\left|\nu_{i}\right|}\left(U_{i, .}^{n}-\min \left(U_{i, .}^{n}, U_{i+1, .}^{n}\right)\right)
\end{array}\right.
$$

3. if $\nu_{i} \leq 0$ ans $\nu_{i+1} \geq 0$, then

$$
F_{i+\frac{1}{2}}^{L}=U_{i, .}^{n} \text { and } F_{i+\frac{1}{2}}^{R}=U_{i+1,}^{n}
$$

4. last, if $\nu_{i} \nu_{i+1}>0$ then

$$
\begin{cases}F_{i+\frac{1}{2}}^{R}=F_{i+\frac{1}{2}}^{L} & \text { if } \nu_{i}>0, \\ F_{i+\frac{1}{2}}^{L}=F_{i+\frac{1}{2}}^{R} & \text { if } \nu_{i+1}>0\end{cases}
$$

Last, the solution of the HJB equation (2.9)-(2.10) on the cell $M_{I}$ is then obtained following

$$
V_{I}^{n+1}=\max \left(W_{I}, \min _{\substack{\lambda^{d} \in \mathcal{S}\left(\xi_{I}\right) \\ a^{d} \in \mathcal{A}^{d}}} U_{I}^{n+1}\left(a^{d}, \lambda^{d}\right)\right)
$$

\subsection{Optimal trajectories reconstruction}

The optimal trajectory reconstruction is obtained by minimizing the time function along possible trajectories (see [2], Appendix by Falcone). It is then necessary to know the minimal time function $\mathcal{T}$ on all over the domain $\mathcal{D}$ and not only around the front at the final time. In practice, the values of $\mathcal{T}$ are saved during the computation of the solution $v$ of (2.9)-(2.10). We follow the process proposed in [6]. Thus, once we have solved the HJB equation (2.9)-(2.10), we get the reachability function $v$ and we use (2.11) to reconstruct $\mathcal{T}$ from $v$. A numerical approximation $\mathcal{T}_{I}$ of $\mathcal{T}\left(\xi_{I}\right)$ is computed as follows. We first set $\mathcal{T}_{I}$ equal to a large numerical value everywhere on the domain $\mathcal{D}$. Then, as the computation of $V_{I}^{n}$ goes on, for $n=0$ up to $n=N-1$, ( $N$ being the number of iterations in time) we consider that if

$$
\left(V_{I}^{n}>0, V_{I}^{n+1}=0 \text { and } \mathcal{T}_{I}^{n}=\infty\right)
$$

then we set $\mathcal{T}_{I}^{n+1}:=t^{n+1}$.

In other words, $\mathcal{T}_{I}$ is the first time when the value of the cell $M_{I}$ goes to 0 , otherwise $\mathcal{T}_{I}=\infty$.

\section{Numerical Simulations in $4 \mathrm{D}$}

This section is devoted to the evaluation from a numerical point of view of the HJB approach proposed in this paper. So, we present 4-dimensional numerical simulations for different scenarios corresponding to configurations arising in air traffic management area and we show how the method allows both to take into account wind current effects, fixed obstacles and conflict avoidance.

For each scenario, we investigate optimal trajectories reconstruction for a two-aircraft configuration in the presence or not of constraints. 


\subsection{D numerical method validation}

This first part is devoted to the validation of the numerical method applied to discretize the HJB equation (2.9). We consider here two simple configurations for which the behavior of the solution is known a priori. still follow a straight line trajectory but are at the same position $(0,0)$ at the same time. We then consider that the $4 \mathrm{D}$ numerical method is validated.
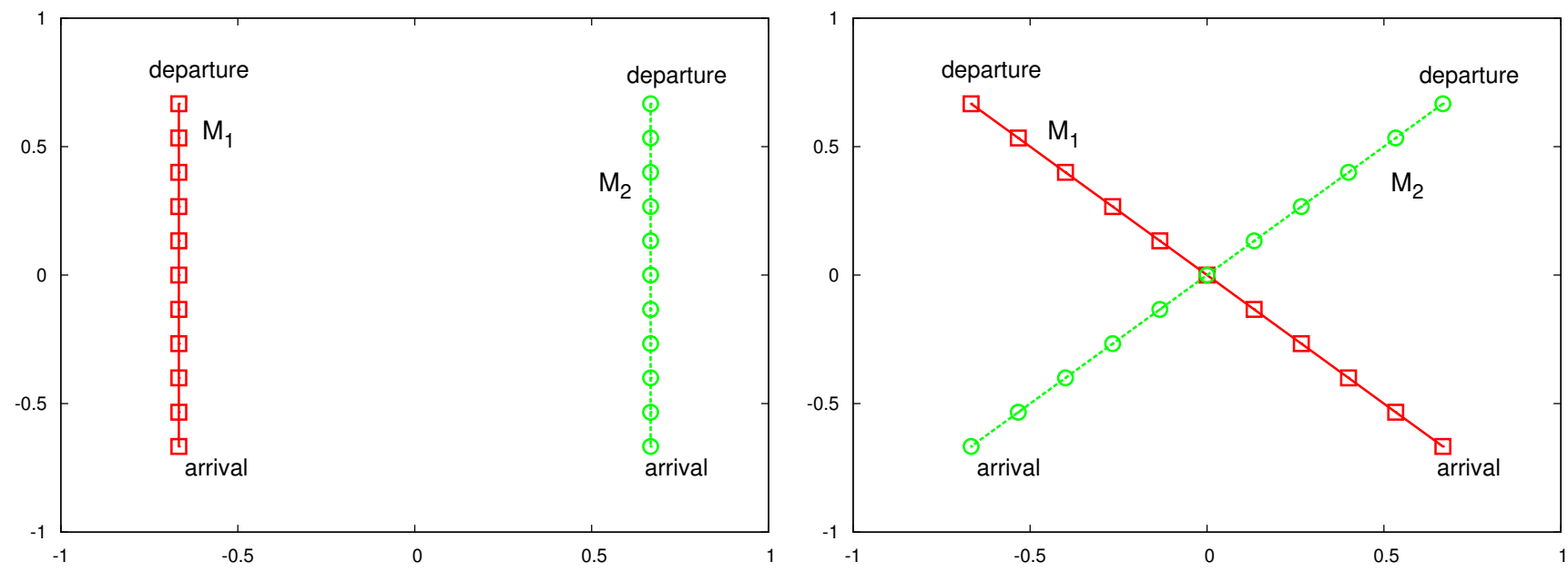

Figure 1: Optimal trajectories for two aircraft with no wind and no obstacles: no conflict case (left side) and conflict configuration (right side)

\subsection{Optimal trajectories under wind effect}


In this scenario, we consider two aircraft $M_{1}$ and $M_{2}$ each one moving in a square domain $[-1: 1] \times[-1: 1]$. Since we are interested in validation and wind effect, we do not consider any interaction, that means no conflict, between the two aircraft neither fixed obstacle in the computational domain. The first aircraft $M_{1}$ is going from its departure position $(0.7,0.2)$ to the arrival one $(-0.7,0.2)$ and respectively for the second aircraft $M_{2}$ going from $(-0.7,-0.2)$ to $(0.7,-0.2)$. The wind current is such that $V=0.5, u(x, y)=-0.2 * y$ and $v(x, y)=0$ in system (4.15). Thus, wind is going from east to west in the upper part of the domain, growing gradually from $y=0$ to the North direction and symmetrically, wind is going from west to east in the lower part of the domain, growing gradually from $y=0$ to the South direction. Figure 2 presents the results obtained with the HJB system (2.9) with $\mathcal{D}=[-1,1]^{2} \times[-1,1]^{2}$ discretized with the Ultra-Bee scheme presented in Section 3.1. We use 15 points in each space direction, 10 values of control and the time step is satisfying the CFL defined by (3.13). We plot the trajectories reconstructed following the method described in Subsection 3.2 and then smoothed. We first note that the method leads to the optimal trajectory for each aircraft. As expected according to the analytic solution of the Zermelo problem in $2 D$, reconstructed trajectories follow a parabolic curve. Moreover, symmetry in wind definition leads to symmetry in reconstructed trajectories (more or less depending on the smoothing option as also expected). This allows to validate the proposed approach taking into account several mobiles.

Now, looking at the effect of the wind, we can observe that trajectories are deviating from the no-wind straight lines ones (plotted in dash lined on Figure 2) to take advantage of the wind. Let us note that the longer distance is counterbalanced by the higher ground speed of the aircraft such that the arrival time is less optimal in the presence of wind.

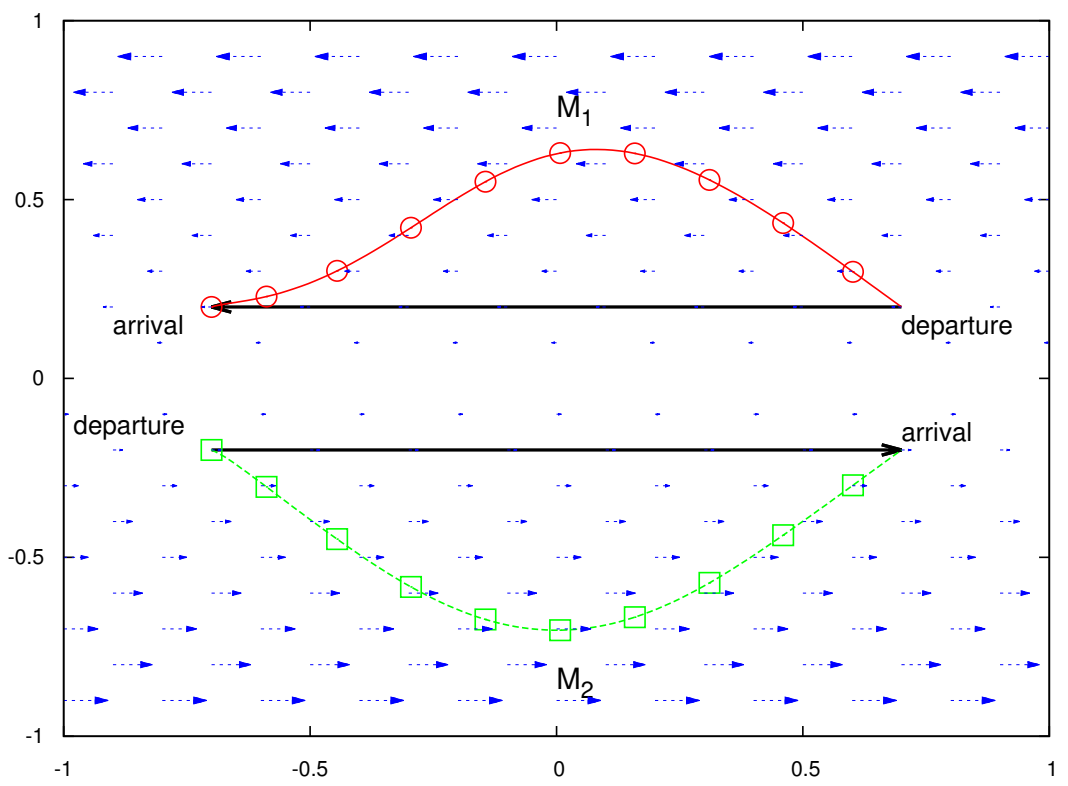

Figure 2: Optimal trajectories for two aircraft under wind effect. 


\subsection{Optimal trajectories under wind effect taking into account fixed obstacles}

In this part, we will consider a more complex scenario composed of two aircraft flying through wind current with some fixed obstacles to avoid. From an operational point of view, these fixed obstacles can described for instance dangerous meteorological areas or areas for which flying over is forbidden. Here, the computational domain is $\mathcal{D}=[-1.2,1.2]^{2} \times[-1.2,1.2]^{2}$. The first aircraft $M_{1}$ is going from its departure position $(-0.96,-0.48)$ to the arrival one $(0.96,0.96)$ and respectively for the second aircraft $M_{2}$ going from $(-0.48,-0.96)$ to $(0.96,0.48)$. The wind current we consider here is similar to the one used for scenario 1 except that it moves in the opposite direction with $V=1, W_{x}=-0.5 * y$ and $W_{y}=0$ in system (2.2). The obstacles are defined by circles respectively centered in $(0.4,0.56),(0.4,-0.56)$ and $(-0.56,-0.56)$ with radius equal to 0.16 . We use 15 points in each space direction, 20 values of control and the time step is satisfying the CFL defined by (3.13).

Figure 3 presents the results obtained in two cases. First we plot the reconstructed trajectories taking into account obstacles without wind, this corresponds to the trajectories plotted in green line for aircraft $M_{1}$ and in red line for aircraft $M_{2}$. Next, we plot the reconstructed trajectories with wind and obstacles in green line with circles for aircraft $M_{1}$ and respectively in red line with squares for aircraft $M_{2}$. Let us note that once again in order to evaluate the potential of the HJB approach to take into account obstacles, departure and arrival positions for the aircraft have been chosen in order that no conflict happens in this scenario. We can first observe that the method well performs since the fixed obstacles are avoided by the aircraft. Moreover, we show that the two trajectories do not behave in the same way to get around the obstacles due to the wind direction. Indeed, the trajectory for aircraft $M_{1}$ takes more advantage of the wind while avoiding obstacles and we can note that wind-optimal trajectories can be far from the optimal trajectories computed without wind.

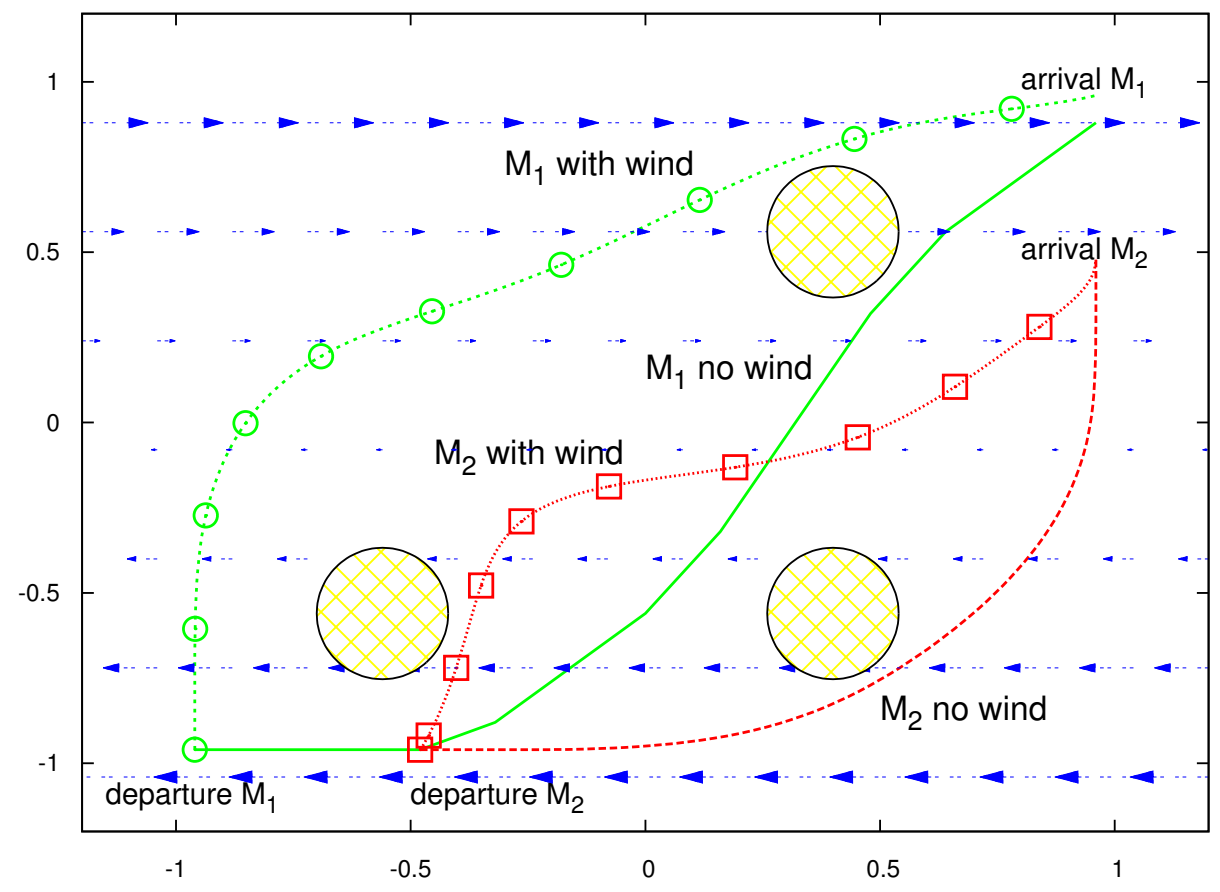

Figure 3: Optimal trajectories for two aircraft taking into account fixed obstacles under wind effect. 


\subsection{Optimal trajectories with conflict avoidance}

In this subsection, we are now interested in evaluating the potential of the proposed approach to treat conflict avoidance. For this purpose, we will study three cases: a first one with no wind and no obstacle (see

FigureTest3), a second one with wind effect and no obstacle (see FigureTest4) and the last one with no wind and obstacle (see FigureTest4).

\subsubsection{Optimal trajectories with conflict avoidance, no wind and no obstacle}

Once again we consider a two-aircraft configuration and in order to dissociate different effects, we consider here that there is no wind current. The first aircraft $M_{1}$ is going from its departure position $(-0.7,0.7)$ to the arrival one $(0.7,-0.7)$ and respectively for the second aircraft $M_{2}$ going from $(-0.7,-0.7)$ to $(0.7,0.7)$. This configuration can illustrate for instance aircraft that are just landing and are going to park in an assigned place around the airport. These positions have been chosen such that if the constraint on conflict avoidance defined by (2.5) is not included in the model then the two-aircraft will be in conflict at point $(0,0)$. This is illustrated with the dashed lines of Figure 4 left side. The dynamics for each aircraft is such that $\left.\dot{(} X_{i}(t)\right)=\left(\cos \left(\theta_{i}\right)\right.$, $\left.\sin \left(\theta_{i}\right)\right)$. We use 15 points in each space direction and 10 control values in $[0,2 \pi]$ while the time step satisfies the CFL condition. In Figure 4, we plot the global trajectories (left side) obtained taking into account the conflict avoidance conflict in the model for each aircraft (blue line with circles for aircraft $M_{1}$ and magenta line with squares for aircraft $M_{2}$ ). Each circle or square correspond to a time, the 4D trajectory has been evaluated with the reconstruction process. In order to verify that with the conflict avoidance constraint, trajectories do not cross, we plot a zoom on the conflict area in Figure 4 (right side) with the corresponding times and we show that aircraft are never at the same position in the same time in the $4 D$ configuration space as required.
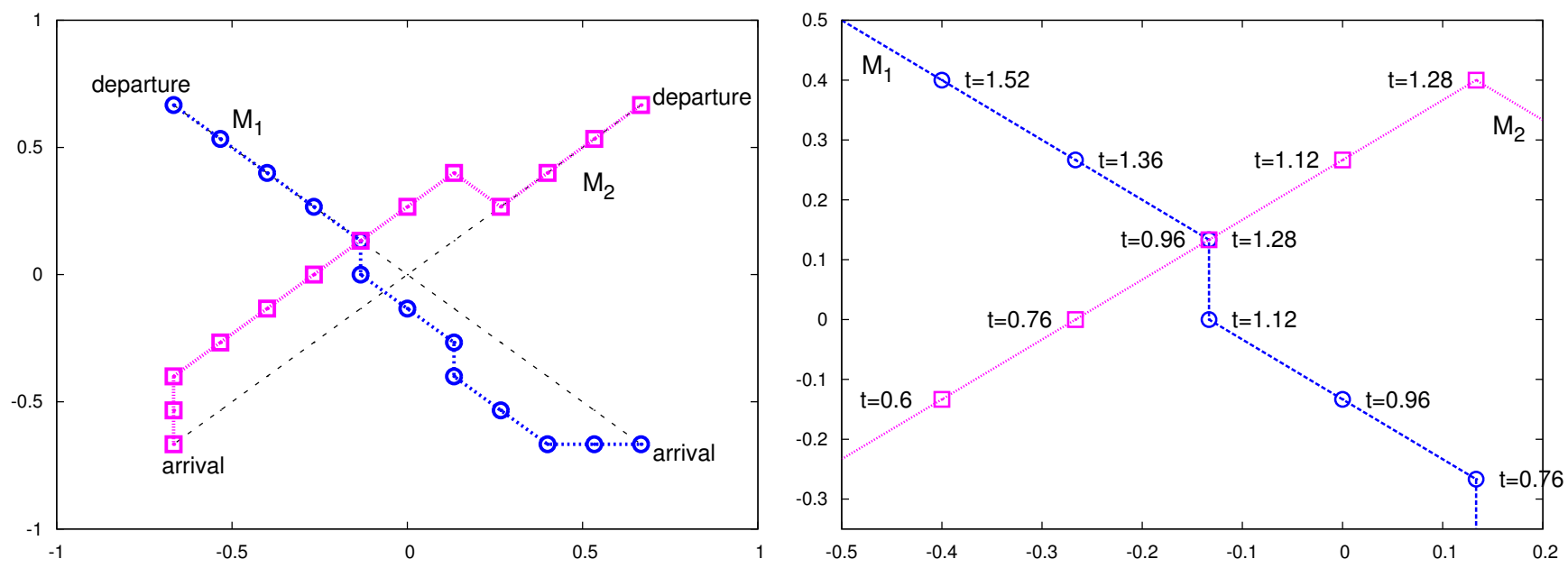

Figure 4: Optimal trajectories for two aircraft taking into account conflict avoidance. Global trajectories (left side) and zoom on the conflict zone (right side).

\subsubsection{Optimal trajectories with conflict avoidance taking into account wind}

In order to evaluate the relevance of the method proposed in this work, we now study a new configuration adding wind effect. Aircraft initial positions are identical with those defined in previous section 4.4.1.However, 
this time the dynamic for each aircraft is given by $\left.\dot{(} X_{i}(t)\right)=\left(-0.2 * x_{2}+0.5 * \cos \left(\theta_{i}\right), \sin \left(\theta_{i}\right)\right)$. In Figure 5 we plot trajectories without conflict avoidance on the left side and with conflict avoidance on the right one and once again, we demonstrate the potential of the method to avoid conflict in the presence of wind.
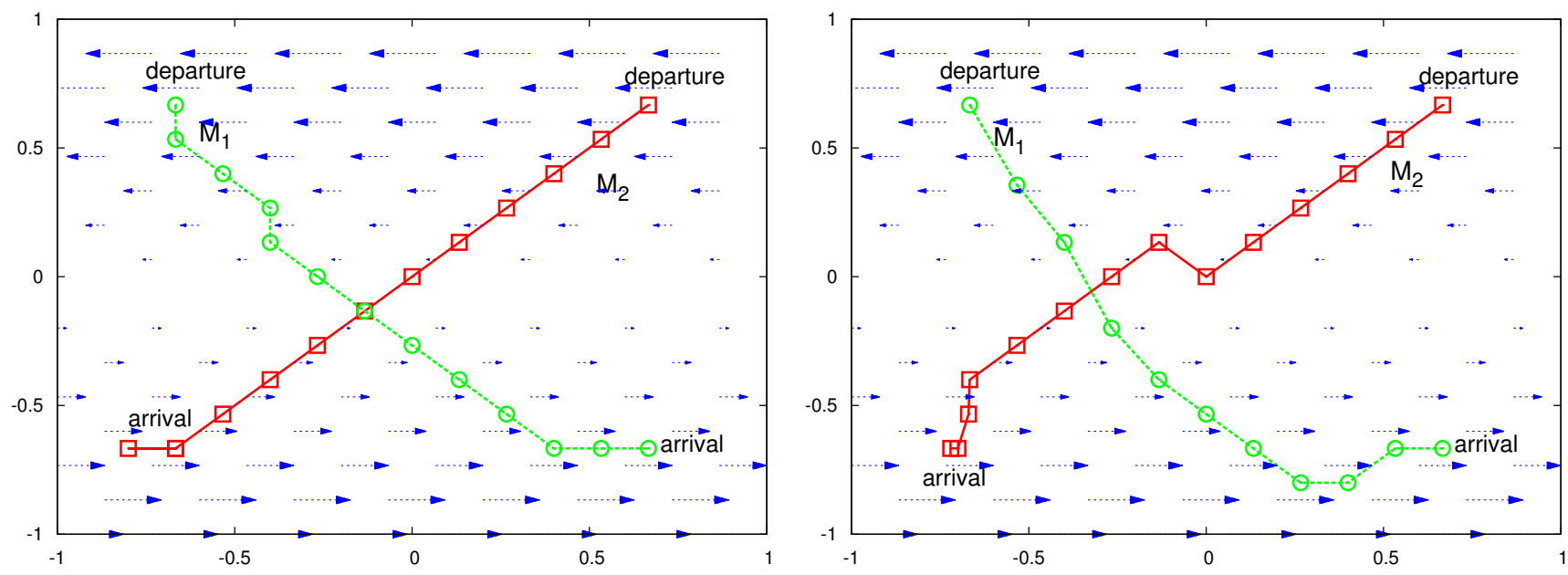

Figure 5: Optimal trajectories for two aircraft taking into account conflict avoidance under wind effect. Trajectories in conflict (left side) and trajectories when the conflict avoidance constraint is included in the model (right side).

\subsubsection{Optimal trajectories with conflict avoidance taking into account fixed obstacles}

The last test concerns conflict avoidance in the presence of fixed obstacle. We still use the computational domain and the departure and arrival positions of the two previous cases for aircraft. Wind is not consider in this test but we add a squared fixed obstacle of size 0.3 centered at $(0,0)$. the dynamics for each aircraft is $\left.\dot{(} X_{i}(t)\right)=\left(\cos \left(\theta_{i}\right), \sin \left(\theta_{i}\right)\right)$ with the same numerical parameters as in section 4.4.1. As previously, we plot in Figure 6 trajectories without conflict avoidance on the left side and with conflict avoidance on the right one and once again and show that the method is also able to avoid conflict in the presence of fixed obstacles.
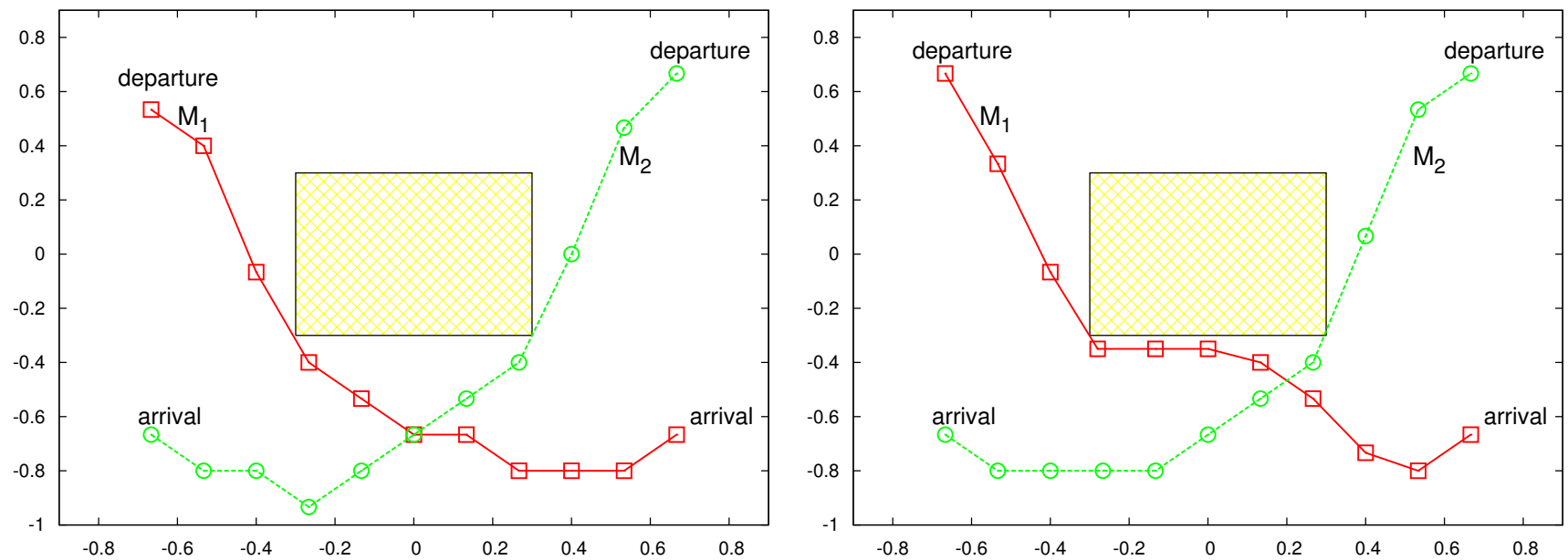

Figure 6: Optimal trajectories for two aircraft taking into account fixed obstacles and conflict avoidance. Trajectories in conflict (left side) and trajectories when the conflict avoidance constraint is included in the model (right side). 


\section{Conclusion and perspectives}

In this paper, we propose a new framework based on optimal control and Hamilton Jacobi Bellman approach for trajectories planning. The model we develop is able to take into account for several mobiles, conflict avoidance, wind current and fixed obstacles bypassing. We use the Ultra-Bee numerical scheme in order to discretize the $4 D$ space domain plus time and present several numerical results that demonstrate the potential of the proposed approach for different two aircraft configurations. Compared to others existing methods, the main advantage of this approach is that it provides a global optimal solution without initialization and convergence questions. However, it has to faced the so-called curse of dimensionality. As a consequence a possible future work could be to extent the model to a configuration involving a larger number of mobiles taking advantage of recent developments of efficient algorithms to solve HJB equation. Another future direction to increase the number of aircraft taken into account in the planning, could concern the possibility to initialize PMP (Pontryagin Minimum Principle Method) using the trajectories resulting from the HJB approach. Indeed, following the method proposed in [17], a rough approximation of the value function computed by the HJB approach can be used to obtain an initial guess for the PMP method. In this case, due to the properties of the HJB equations, the main advantage is that the initial guess will be close to the global minimum.

\section{References}

[1] K. Alton. Dijkstra-like Ordered Upwind Methods for Solving Static Hamilton-Jacobi Equations. PhD thesis, The University of British Columbia, Vancouver, Canada, (2010).

[2] M. Bardi and I. Capuzzo-Dolcetta, Optimal control and viscosity solutions of Hamilton-Jacobi-Bellman equations. Systems and Control: Foundations and Applications. Birkhauser, Boston, (1997).

[3] R.E. Bellman, Dynamic Programming. Princeton University Press, (1957).

[4] J.T. Betts, Practical methods for optimal control using nonlinear programming. Society for Industrial and Applied Mathematics, (2001).

[5] O. Bokanowski, N. Forcadel, H. Zidani, Reachability and minimal times for state constrained nonlinear problems without any controllability assumption, SIAM J.Control Optim., 48 (2010), pp. 4292-4316.

[6] O. Bokanowski, A. Briani, H. Zidani, Minimum time control problems for non autonomous differential equations, Systems \& Control Letters, 58 (2009), pp. 742-746.

[7] O. Bokanowski and H. Zidani, Anti-diffusive schemes for linear advection and application to HamiltonJacobi-Bellman equations, J. Sci. Comput., 30 (2007), pp. 1-33.

[8] O. Bokanowski, S. Martin, R. Munos, and H. Zidani. An anti-diffusive scheme for viability problems. Applied Numerical Mathematics, 56:1147-1162, (2006).

[9] O. Bokanowski, E. Bourgeois, A. Désilles, H. Zidani, Optimization of the launcher ascent trajectory leading to the global optimum without any initialization: the breakthrough of the HJB approach. Proceeding Eucass 2015 - 6th European Conference for Aeronautics and Space Sciences, (2015). 
[10] O. Bokanowski, E. Cristiani, J. Laurent-Varin and H. Zidani, Hamilton-Jacobi-Bellman approach for the climbing problem for multi-stage launchers, pp. 8 pages, 20th International Symposium on Mathematical Theory of Networks and Systems (MTNS'2012), (2012).

[11] O. Bokanowski, Y. Cheng, C.-W. Shu, A discontinuous Galerkin scheme for front propagation with obstacles, Numerische Mathematik, Volume 126, Issue 1, pp 1-31, (2014).

[12] A.E. Bryson, Y-C. Ho, Applied Optimal Control: Optimization, Estimation and Control, Taylor \& Francis, (1975).

[13] S. Cacace, E. Cristiani, M. Falcone,Can local single-pass methods solve any stationnary Hamilton-JacobiBellman equation? SIAM J.Sci.Compt. 36,A570-A587, (2014).

[14] E. Carlini, M. Falcone, and R. Ferretti. An efficient algorithm for Hamilton-Jacobi equations in high dimensions. Comput. Vis. Sci., 7:15-29, (2004).

[15] S. Chaimatanan, D. Delahaye, M. Mongeau. Strategic Deconfliction of Aircraft Trajectories. In ISIATM 2013, 2nd International Conference on Interdisciplinary Science for Innovative Air Traffic Management, (2013).

[16] Cheng, Y., Shu, C.-W.: A discontinuous Galerkin finite element method for directly solving the HamiltonJacobi equations. J. Comput. Phys. 223, 398-415 (2007).

[17] E. Cristiani and P. Martinon, Initialization of the shooting method via the Hamilton-Jacobi-Bellman approach, J. Optim. Theory Appl., 146 (2010), pp. 321-346.

[18] M. Crandall and P.-L. Lions. Two approximations of solutions of Hamilton Jacobi equations. Mathematics of Computation, 43:1-19, (1984).

[19] E. Cruck, A. Désilles and H. Zidani, Collision analysis for a UAV, AIAA Guidance, Navigation and Control Conference, Minneapolis (USA), 13-16 August, vol. Chapt. 4526, pp. 23 pages, (2012).

[20] D. Delahaye, S. Puechmorel, E. Feron, P. Tsiotras, Mathematic Models for Aircraft Trajectory Design: A Survey, 2013 ENRI International Workshop on ATM/CNS (EIWAC2013). Tokyo, Japan, (2013).

[21] B. Desprès, F. Lagoutière, Un schéma non linéaire anti-dissipatif pour l'équation d'advection linéaire. A non-linear anti-diffusive scheme for the linear advection equation. C. R. Acad. Sci., Paris, Sér. I, Math. 328, 939-944 (1999).

[22] B. Desprès, F. Lagoutière. Contact discontinuity capturing schemes for linear advection and compressible gas dynamics. J.Sci. Comput., 16:479-524, (2001).

[23] N. E. Dougui, D. Delahaye, S. Puechmorel, M. Mongeau. A light-propagation model for aircraft trajectory planning. Journal of Global Optimization, Springer Verlag, 56 (3), (2013), pp 873-895.

[24] M. Falcone, R. Ferretti. Semi-Lagrangian schemes for Hamilton-Jacobi equations, dis- crete representation formulae and Godunov methods. Journal of Computational Physics, 175:559-575, (2002). 
[25] M. Falcone, R. Ferretti. Semi-Lagrangian approximation schemes for linear and Hamilton-Jacobi equations, SIAM (2014).

[26] T.Schouwenaars, J. How, E. Feron, Decentralized Cooperative Trajectory Planning of Multiple Aircraft with Hard Safety Guarantees, IAA Guidance, Navigation, and Control Conference and Exhibit 16 - 19 August 2004, Providence, Rhode Island.

[27] L. Guys, S. Puechmorel,L. Lapasset,Automatic conflict solving using biharmonic navigation functions, EWGT2012, 15th Meeting of the EURO Working Group on Transportation, Sep 2012, Paris, France. Elsevier, 54 (4), pp 1378-1387, (2012).

[28] K. Margellos and J. Lygeros. Hamilton-Jacobi formulation for Reach-Avoid Problems with an application to Air Traffic Management. In American Control Conference, Baltimore, MD, USA, (2010).

[29] I. M. Mitchell, A. Bayen, C. J. Tomlin, A Time-Dependent Hamilton-Jacobi Formulation of Reachable Sets for Continuous Dynamic Games, IEEE Transactions on Automatic Control (Volume 50, Issue 7):947-957, 2005.

[30] A. Oikonomopoulos, S. Loizou, K. Kyriakopoulos. Coordination of multiple non-holonomic agents with input constraints. In IEEE International Conference on Robotics and Automation (ICRA09), pages 869$874,(2009)$.

[31] S. Osher and C.-W. Shu. High order essentially non-oscillatory schemes for Hamilton-Jacobi equations. SIAM J. Numer. Anal., 28:907-922, (1991).

${ }_{425}$ [32] J.J. Pannequin, A.M. Bayen, I.M. Mitchell, H. Chung, S. Sastry. Multiple aircraft deconflicted path planning with weather avoidance constraints. In Proceedings of AIAA Guidance, Navigation and Control Conference, (2007).

[33] D. Sislak. Agent-Based Approach to Air-Traffic Modeling, Simulation and Collision Avoidance. PhD thesis, Czech Technical University, (2013).

[34] Y.-T. Zhang, C.-W. Shu. High order WENO schemes for Hamilton-Jacobi equations on triangular meshes. SIAM J. Sci. Comput., 24:1005-1030, (2003).

[35] T. Zheng, X. Zhao. A Novel Approach for Multiple Mobile Robot Path Planning in Dynamic Unknown Environment. In IEEE Conference on Robotics, Automation and Mechatronics, pages 1-5, (2006). 